\title{
Postnatal development of the astrocyte perivascular MLC1/GlialCAM complex defines a temporal window for the gliovascular unit maturation
}

Alice Gilbert ${ }^{1}$, Xabier Elorza Vidal ${ }^{2,3}$, Raul Estevez ${ }^{2,3}$, Martine Cohen-Salmon ${ }^{1}$ and Anne-Cécile Boulay ${ }^{1}$

${ }^{1}$ Collège de France, Center for Interdisciplinary Research in Biology (CIRB)/ Centre National de la Recherche Scientifique CNRS, Unité Mixte de Recherche 7241/ Institut National de la Santé et de la Recherche Médicale INSERM, U1050 / 75231 Paris Cedex 05, France ; Paris Science Lettre Research University, 75005 Paris, France.

${ }^{2}$ Unitat de Fisiología, Departament de Ciències Fisiològiques, IDIBELL-Institute of Neurosciences, Universitat de Barcelona, L'Hospitalet de Llobregat, Spain.

${ }^{3}$ Centro de Investigación en Red de Enfermedades Raras (CIBERER), ISCIII, Spain.

Corresponding author: Martine Cohen-Salmon, Collège de France, Center for Interdisciplinary Research in Biology (CIRB), CNRS, UMR 7241, INSERM, U1050, 11 place Marcelin Berthelot Paris, 75005, France. Tel: +33- 144271242 ; Fax : +33- 144271268 ; E-mail : martine.cohen-salmon@ college-de-france.fr 
Astrocytes, the most abundant glial cells of the central nervous system are morphologically complex. They display numerous processes interacting with synapses and blood vessels. At the vascular interface, astrocyte endfeet-terminated processes almost entirely cover the blood vessel surface and participate to the gliovascular unit where important vascular properties of the brain are set such as the blood-brain barrier (BBB) integrity. How specific morphological and functional interactions between astrocytes and the vascular compartment develop has not been fully investigated. Here, we elaborated an original experimental strategy to study the postnatal development of astrocyte perivascular endfeet. Using purified gliovascular units, we focused on the postnatal expression of MLC1 and GlialCAM, two transmembrane proteins forming a complex enriched at the junction between mature astrocyte perivascular endfeet. We showed that MLC1 and GlialCAM were enriched and assembled into mature complexes in astrocyte perivascular endfeet between postnatal days 10 and 15, after the formation of astrocyte perivascular Aquaporin 4 water channels. These events correlated with the increased expression of Claudin-5 and PgP, two endothelial-specific BBB components. These results illustrate for the first time that astrocyte perivascular endfeet differentiation is a complex and progressive process which correlates with BBB maturation. Moreover, our results suggest that maturation of the astrocyte endfeet MLC1/GlialCAM complex between postnatal days 10 and 15 might be a key event in the gliovascular unit maturation.

Keywords

Astrocyte; Gliovascular unit; Blood-brain barrier; Postnatal development; MLC1; GlialCAM

\section{Acknowledgments}

This work has been financed by FRM "Fondation pour la recherche médicale" (PLP20170939025p60 to AG, AJE20171039094 to MCS and SPF20150934067 to ACB), "Fondation Maladies Rares", the European Leukodystrophies Association (ELA) Research Foundation (ELA2012-014C2B to RE), the Spanish Ministerio de Ciencia e Innovación (MICINN) (SAF2015-70377 to RE), the Generalitat de Catalunya (SGR2014-1178 to 
RE). RE is a recipient of an ICREA Academia prize. We thank Anna Capano for technical help and Philippe Mailly for help in image analysis. We are grateful to Xavier Declèves and Jean-Louis Laplanche for helpful discussions and constant support.

\section{Author Contributions}

MC-S and A-CB designed research; AG and A-CB performed research; AG, MC-S and A-CB analyzed data; XEV and RE provided resources; A-CB and MC-S wrote the paper. Authors declare no conflicts of interest. 


\section{Introduction}

Astrocytes are glial cells now recognized as key actors of the brain physiology (Vasile et al. 2017; Allen and Eroglu 2017). They closely interact with both the neuronal and the vascular compartments, maintaining and modulating synaptic and brain vascular functions. At the vascular interface, astrocytes are involved in the coupling between blood flow and neuronal activity (Attwell et al. 2010). They maintain blood-brain barrier (BBB) integrity by contributing to the basal lamina composition (Menezes et al. 2014) or controlling the expression of endothelial tight junction proteins and transporters (Abbott et al. 2006). They regulate perivascular homeostasis and fluid circulation (Amiry-Moghaddam et al. 2004; Iliff et al. 2012). They play a key role in brain immune quiescence, controlling the recruitment and fate of immune cells and influencing inflammatory processes in the brain (Alvarez et al. 2013). Astrocyte regulation of brain vascular functions is sustained by a morphological and molecular polarity. At the vascular interface, they send processes called endfeet which almost entirely cover the brain vasculature and form together with endothelial and mural cells (pericytes or smooth muscle cells) the gliovascular unit (GVU) (Mathiisen et al. 2010). They are equipped with a molecular repertoire dedicated to the regulation of the vascular system, which includes the gap junction proteins Connexin 30 and 43 (Boulay et al. 2015b, a) and the water channel Aquaporin 4 (Aqp4) (Amiry-Moghaddam et al. 2004; Hawkes et al. 2011). How this specialized astrocyte interface with the vascular system is set during development is unknown. Vessels are formed during embryonic development from a perineural vascular plexus. They penetrate the neuroectoderm and form immature blood vessels. Around embryonic day (E) 15 in the mouse, neighboring cells such as pericytes induce a primitive endothelial BBB (Ben-Zvi et al. 2014; Chen et al. 2017). At birth, while angiogenesis is mainly completed, acquisition of brain-specific vascular functions continues, in particular BBB maturation (Obermeier et al. 2013) and neurovascular coupling (Schmithorst et al. 2015). Astrocytes are generated from radial glia around E17 and astrocytogenesis continues in the neonates, mainly by local division (Ge et al. 2012; Nagao et al. 2016; Jiang and Nardelli 2016). Astrocyte maturation is achieved by integration within their environment in particular through the formation of polarized processes interacting with blood vessels (Stogsdill et al. 2017). Based on the expression of Aqp4, previous studies suggested that astrocyte endfeet perivascular coverage starts around E18 in the mouse and is complete at P5 in the cortex (Ezan et al. 2012) and around P13 in pial vessels (Lunde et al. 2015). However, a precise description of how and when astrocyte endfeet acquire their molecular repertoire and their specific functions is still lacking.

To address this question, we focused here on MLC1 and GlialCAM, two membrane proteins forming a junctional complex enriched between astrocyte perivascular endfeet in the adult brain (Teijido et al. 2007; 
Hoegg-Beiler et al. 2014). Mutations in their associated genes are linked to Megalencephalic Leukoencephalopathy with subcortical Cysts (MLC), a rare type of leukodystrophy characterized by progressive macrocephaly, that leads to the degradation of motor functions and mental retardation (van der Knaap et al. 2012). In the brain, MLC1 is exclusively expressed by astrocytes and enriched in perivascular endfeet. It has been shown to play a role in osmotic regulations either directly or indirectly through its interaction with other proteins such as the chloride channel CLC-2 (Sirisi et al. 2014). Despite MLC1 similarity with ion channels and transporters, its function is yet unknown. GlialCAM is a member of the immunoglobulin superfamily of cellular adhesion proteins and is expressed in the brain by astrocytes and oligodendrocytes (Chung Moh et al. 2005; Favre-Kontula et al. 2008). It interacts directly with MLC1 and is crucial for its compartmentalization at the astrocyte membrane (López-Hernández et al. 2011b; Jeworutzki et al. 2012). It also protects MLC1 from degradation by acting as a chaperone (Capdevila-Nortes et al. 2013a). Previous reports showed that MLC1 expression starts around E16 in the mouse brain, mainly in progenitor cells from the ventricular zone, and increases specifically in astrocytes until 3 weeks postnatal (Schmitt et al. 2003), while GlialCAM expression increases from birth (Bugiani et al. 2017).

Here, taking advantage of our recently described GVU purification technique (Boulay et al. 2015c, 2017), we characterized the astrocyte perivascular endfeet MLC1 and GlialCAM expression during postnatal development. We show that GlialCAM precedes MLC1 expression and that both proteins increase in already formed Aqp4expressing astrocyte perivascular endfeet between P10 and P15, in parallel to the BBB maturation. Our results provide the first evidence that astrocyte endfeet development is a stepwise process with sequential acquisition of a polarized molecular repertoire. 


\section{Material and methods}

\section{Mice}

OF1 mice were purchased from Janvier Labs. (France) and hosted in pathogen free conditions. Male and female were equally included in the study.

\section{Ethical approval}

All applicable international, national, and institutional guidelines for the care and use of animals were followed. All procedures performed were in accordance with the ethical standards of the institution at which the studies were conducted.

\section{Gliovascular unit purification}

Gliovascular units (GVU) were isolated from whole brains of OF1 mice as previously described (Yousif et al. 2007; Boulay et al. 2017). Selective filtration was performed with a negative filtration using a $100 \mu \mathrm{m}$ nylon mesh filter to remove meninges, choroid plexus and periventricular layers, followed by a positive filtering on a $20 \mu \mathrm{m}$ nylon mesh filter to retain parenchymal vessels and remove debris from other brain cell types.

\section{Western-blot}

Proteins were extracted from purified GVUs ( 5 brains pooled per sample) or brains ( 1 hemisphere per sample) in respectively $50 \mu \mathrm{L}$ and $500 \mu \mathrm{L}$ of $2 \%$ SDS per sample with EDTA-free Complete Protease Inhibitor (Roche, Mannheim, Germany), sonicated three times at $20 \mathrm{~Hz}$ (Vibra cell VCX130, Sonic \& Materials, Newtown, USA) and centrifuged $20 \mathrm{~min}$ at $10000 \mathrm{~g}$ at $4{ }^{\circ} \mathrm{C}$. Supernatants were heated in Laemmli loading buffer for $5 \mathrm{~min}$ at 56

${ }^{\circ} \mathrm{C}$. Three independent samples were prepared for each developmental stage $(n=3)$.Protein content was measured using the Pierce $660 \mathrm{~nm}$ protein assay reagent (Thermo Scientific, Rockford, IL, USA). $20 \mu \mathrm{g}$ of proteins were separated by denaturing electrophoresis in 4-12\% NuPAGE gradient gel (or 3-8\% Tris Acetate for the P-gP) (Thermo Fisher, Waltham, MA, USA) and electrotransferred to nitrocellulose membranes. Membranes were analyzed as previously described (Boulay et al. 2017). HRP activity was visualized by ECL using Western Lightning plus enhanced chemoluminescence system (Perkin Elmer, Waltham, MA, USA) on a LAS4000 (Fujifilm, Tokyo, Japan). $\beta$-Actin content was used as a loading reference. Semi-quantitative densitometric analysis was performed with ImageJ software after scanning the bands.

P5 to P15 and P15 and P60 samples were loaded and analyzed separately. Global change in expression was tested using the non parametric test Kruskal-Wallis (indicated as p-value $\mathrm{KW}_{\mathrm{W}}$ ), and between each stage using the Mann-Whitney (indicated as p-value $\mathrm{P}_{\mathrm{P} / \mathrm{P} 10}$ to $\mathrm{p}$-value $\mathrm{P}_{\mathrm{P} 15 / \mathrm{P} 60}$ ). Data are represented as mean $\pm \mathrm{sd}$. 
qPCR

mRNAs from mice hemispheres were purified using the RNeasy Lipid tissue kit (Qiagen). cDNA were synthesized from $1 \mu \mathrm{g}$ RNA using Reverse Transcriptase Superscript III kit with random primers and stored at $20^{\circ} \mathrm{C}$. PCR was performed following the droplet PCR procedure (Bio-Rad; Hercules, CA, USA) in 20- $\mu \mathrm{L}$ reactions using ddPCR Supermix for Evagreen (Bio-Rad; Hercules, CA, USA) on $1 \mu \mathrm{L}$ of cDNA diluted in water 1/6 for Mlc1 and Glialcam (Hepacam) and 1/60 for Gapdh. Droplets were generated following manufacturer's instructions. PCR cycling was $95^{\circ} \mathrm{C}$ for $5 \mathrm{~min}$, and 40 cycles of $95{ }^{\circ} \mathrm{C}$ for $30 \mathrm{~s}$ and $60{ }^{\circ} \mathrm{C}$ for 1 min. Primers: Hepacam forward 5'-GACCGCCTAAAATCAGAAGCA -3'; Hepacam reverse 5'TGGCTCTGTAGCAGGGTTTT-3'; Mlc1 forward 5'-TCAGTGCGATTCCCAACTTTCA-3'; Mlc1 reverse 5'GGACCGGGCCGAAATGAT-3'; Gapdh forward 5'-AGGTCGGTGTGAACGGATTTG-3'and Gapdh reverse 5'- TGTAGACCATGTAGTTGAGGTCA-3'. Positive droplets were counted on a QX200 ddPCR droplet reader (Bio-Rad; Hercules, CA, USA) and the QuantaSoft Analysis software (Bio-Rad; Hercules, CA, USA). Mlc1 and Hepacam expression were normalized on Gapdh. Change in expression was tested using the Kruskal-Wallis and Mann-Whitney non-parametric tests. Data are represented as mean \pm sd.

\section{Immunostaining}

Hippocampal slices: mice were anesthetized with Pentobarbital $(600 \mathrm{mg} / \mathrm{kg}$, i.p. $)$ and killed by transcardiac perfusion with PBS/PFA 4\%. Brain was removed and cut in $30 \mu$ m-thick sections using a Leitz (1400) sliding microtome. Three animals were analyzed per developmental stage.

Gliovascular units: GVUs were plated on a glass slide coated with Cell Tak (Corning, Corning, NY, USA) and fixed in PBS/PFA 4\% for $15 \mathrm{~min}$ at room temperature. Three animals were pooled for each developmental stage.

Hippocampal slices or GVUs were immersed in the blocking solution (PBS/NGS 5\%/Triton X-100 0.5\%) for $1 \mathrm{~h}$ at room temperature and incubated with primary antibodies and Isolectin GS-B4 (IB4) diluted in the blocking solution $12 \mathrm{~h}$ at $4{ }^{\circ} \mathrm{C}$. After 3 PBS washes, slices or GVUs were incubated $2 \mathrm{~h}$ at room temperature with secondary antibodies, rinsed in PBS and finally embedded in Fluormount G. They were analyzed using a 63X objective on a Nikon Eclipse Ti microscope (Nikon Instruments, Melville, NY, USA). Proportions of vessels with MLC1 or GlialCAM were analyzed with the Chi-squared test with Yates' continuity correction. Among GlialCAM-positive vessel segments, the proportion also co-stained for MLC1 were analyzed using an ANOVA/ Bartlett's test and a Student's test. Among MLC1-positive vessel segments, the proportion co-stained for GlialCAM was analyzed similarly. Data are represented as mean $\pm \mathrm{sd}$. 


\section{Antibodies}

Primary: polyclonal rabbit anti-Aqp4 (1:500) (Sigma, St Louis, MO, USA); polyclonal rabbit anti-Claudin- 5 (1:250) (Thermo Fisher, Waltham, MA, USA); monoclonal mouse anti-P-gP (1:200) (Enzo Life Sciences, Lausen, Switzerland); monoclonal mouse anti-Connexin 43 (1:500) (BD-Biosciences, Franklin Lakes, NJ, U.S.); horseradish peroxidase (HRP)-coupled $\beta$-actin (1:25000) (Abcam, Cambridge, UK); polyclonal rabbit antiGlialCAM (1:200) and monoclonal mouse anti-MLC1 (1:500) (López-Hernández et al. 2011a); Alexaconjugated Isolectin GS-B4 (IB4) was used to label the blood vessel walls (1:100) (Thermo Fisher, Waltham, MA, USA).

Secondary: Alexa-conjugated goat anti-mouse and anti-rabbit IgG (1:1000) (Molecular Probes, Eugene, OR, USA), HRP-conjugated goat anti-mouse or anti-rabbit IgG (1:2000) (Santa Cruz Biotechnology, Santa Cruz, CA, USA). 


\section{Results}

In the adult brain, MLC1 and GlialCAM are not only enriched in astrocyte perivascular endfeet but also expressed in perisynaptic astrocyte processes (Sakers et al. 2017) and in oligodendrocytes for GlialCAM (Jeworutzki et al. 2012). In addition, during brain development, MLC1 is present in progenitors from the ventricular zone (Schmitt et al. 2003). In order to specifically focus our study on astrocyte perivascular proteins, we used our recently developed purification technique of the gliovascular unit (GVU), i.e. perivascular endfeet attached to brain vessels (Boulay et al. 2015c, 2017) (Fig. 1A). As purification of the GVU has been previously obtained only from adult tissues, we first tested if this technique could be developed on early postnatal brains. We concentrated our study on parenchymal vessels, depleting our samples from pial vessels by differential filtering of the purified vessels (see material and methods) (Yousif et al. 2007). To test the presence of astrocyte perivascular endfeet on purified vessels, we performed Aqp4 immunostaining. Blood vessels were labelled by Isolectin GS-B4 (IB4). Immunostaining on postnatal day (P) 5 GVUs revealed a continuous Aqp4 labelling at the surface of most vessels, as observed in adult (P60) GVUs (Fig. 1B). The proportion of Aqp4-positive GVUs was comparable at all stages (Fig. 1C) (P5:83\%; P10:88\%; P15:96\%; P60:93\%; p-value=0.45). Performing Western-blot quantification on purified GVU protein extracts, we observed a small, although not significant, increase of Aqp4 between P5 and P10 followed by a stabilization from P10 onwards (P5:1.0 $\pm 0.2 ;$ P10:1.7 \pm 0.9 ; $\mathrm{P} 15: 1.8 \pm 0.1 ; \mathrm{P} 60: 1.4 \pm 0.1 ; \mathrm{p}$-value $\mathrm{KW}=0.09$ ) (Fig. 1D and E). The same result was obtained using total brain extracts, consistent with the fact that, in the brain, Aqp4 is mostly present in perivascular astrocyte endfeet (P5:1.0 $\pm 0.1 ; \mathrm{P} 10: 1.5 \pm 0.2 ; \mathrm{P} 15: 1.4 \pm 0.1 ; \mathrm{P} 60: 1.6 \pm 0.2 ; \mathrm{p}$-value $\left._{\mathrm{KW}}=0.06\right)$ (Fig. $1 \mathrm{D}$ and $\left.\mathrm{E}\right)$. To further validate our approach, we tested by Western-blot the copurification of the gap junction channel protein Connexin 43 (Cx43) another protein enriched in astrocyte mature endfeet (Ezan et al. 2012). Cx43 was detected in GVU at all stages (Fig. S1). Collectively, these results indicated that intact GVUs can be purified from the brain as early as P5 and that our purification protocol is a valuable tool to explore the molecular composition of astrocyte perivascular endfeet membranes during the postnatal development.

We next addressed perivascular endfeet expression of MLC1 and GlialCAM during the postnatal development from P5. Western-blot of purified GVU protein extracts showed that GlialCAM expression was already detectable at P5 (Fig. 2A). Its quantity significantly increased between P10 and P15 and remained stable onwards (Fig. 2A and B) (P5:1.0 $\pm 0.2 ;$ P10:1.0 $\pm 0.03 ;$ P15:2.1 $\pm 0.2 ;$ P60:2.6 $\pm 0.4 ; \quad$ p-value KW $=0.002 ; \quad$ value $_{\mathrm{P} 5 / \mathrm{P} 10}=0.4 ; \mathrm{p}$ - value $_{\mathrm{P} 10 / \mathrm{P} 15}=0.05 ; \mathrm{p}$-value $\left.\mathrm{P15/ \textrm {P } 6 0}=0.25\right)$. In contrast to GVUs, GlialCAM detected in total brain 
extracts was stable between P10 and P15 (WB, P5:1.0 $\pm 0.1 ; \mathrm{P} 10: 1.9 \pm 0.2 ; \mathrm{P} 15: 1.9 \pm 0.1 ; \mathrm{P} 60: 2.2 \pm 0.1$; pvalue $_{\mathrm{KW}}=0.02 ; \mathrm{p}$-value $\mathrm{P}_{\mathrm{P} / \mathrm{P} 10}=0.05 ; \mathrm{p}-$ value $_{\mathrm{P} 10 / \mathrm{P} 15}=0.5 ; \mathrm{p}-$ value $\left._{\mathrm{P} 15 / \mathrm{P} 60}=0.2\right)$. The same was observed at the mRNA level. We noticed however an increase from P15 to P60 (qPCR, P5:1.0 $\pm 0.2 ; \mathrm{P} 10: 1.2 \pm 0.2 ; \mathrm{P} 15: 0.9 \pm 0.2$; $\mathrm{P} 60: 1.8 \pm 0.6 ; \mathrm{p}-$ value $_{\mathrm{KW}}=0.003 ; \mathrm{p}-$ value $_{\mathrm{P} 5 / \mathrm{P} 10}=0.2 ; \mathrm{p}-$ value $\left._{\mathrm{P} 10 / \mathrm{P} 15}=0.06 ; \mathrm{p}-\mathrm{value}_{\mathrm{P} 15 / \mathrm{P} 60}=0.03\right) \quad(\mathrm{Fig}$. 2B). These results suggested a distinct temporal regulation of GlialCAM expression between the GVU and the rest of the brain between P10 and P15. We previously found that Aqp4-containing astrocyte endfeet were similarly purified at P10 and P15 (Fig. 1). The increase of GlialCAM level in the GVU between P10 and P15 could thus reflect an increased expression in endfeet or an increased number of GlialCAM-expressing endfeet. To solve this question, we evaluated the proportion of vessels surrounded by GlialCAM-immunolabeled astrocyte endfeet on P10 and P15 hippocampal slices. Blood vessels were labeled by IB4 (Fig. 2C and D). The proportion of IB4-positive vessels showing a perivascular GlialCAM labeling was similar at P10, P15 and P60 (P5:77\%; P10:85\%; $\mathrm{P} 15: 92 \% ; \mathrm{p}$-value $\mathrm{P}_{\mathrm{P} 10 / \mathrm{P} 15}=0.4 ; \mathrm{p}$-value $\left.\mathrm{P}_{\mathrm{P} 15 / \mathrm{P} 60}=0.9\right)$. Since this observation was performed on a restricted part of the brain while GVU protein extracts used for Western-blot were obtained from whole brains, we further excluded the possibility of regional variation by quantifying the proportion of GlialCAM-positive purified GVU. The same proportion was found than on brain sections (Fig. 2E and F) (P5:86\%; P10:90\%; P15:83\%; pvalue $_{\mathrm{P} 5 / \mathrm{P} 10}=0.8 ; \mathrm{p}$-value $\left.\mathrm{P}_{\mathrm{P} 0 / \mathrm{P} 15}=0.2\right)$. Thus, GlialCAM is expressed around most brain vessels at P10 and its expression increases in the GVU between P10 and P15. In addition, while at P10, GlialCAM immunostaining was mainly dispersed, it formed clear meshworks surrounding the vessels from P15, suggesting a maturation of its subcellular localization (Fig. 2E).

In contrast to GlialCAM, Western-blot analysis of MLC1 expression in purified GVUs showed a very weak signal at P5 and P10, which significantly increased at P15 and was stable onwards (Fig. 3A and B) (P5:1.0 \pm 0.1 ;

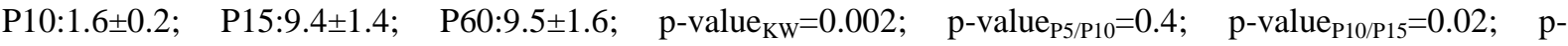
value $\left._{\mathrm{P} 15 / \mathrm{P} 60}=0.9\right)$. This increase was not detected in total brain extracts both by Western Blot and qPCR suggesting that it is an astrocyte endfeet-specific event (WB, P5:1.0 $\pm 0.06 ; \mathrm{P} 10: 1.3 \pm 0.2 ; \mathrm{P} 15: 1.6 \pm 0.1$;

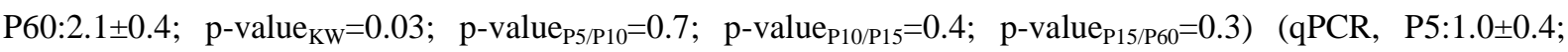
P10:0.9 $\pm 0.1 ; \quad P 15: 0.6 \pm 0.2 ; \quad P 60: 0.9 \pm 0.1 ; \quad p$-value $_{K W}=0.15 ; \quad p-$ value $_{P 5 / P 10}=0.9 ; \quad p-$ value $_{P 10 / P 15}=0.06 ; \quad p-$ value $_{\mathrm{P} 15 / \mathrm{P} 60}=0.06$ ) (Fig. 3B). On hippocampal slices, perivascular MLC1 immunostaining was barely detectable at P10, while highly present in the astrocyte perivascular endfeet at P15 and P60 (P10:20\%; P15:90\%; $\mathrm{P} 60: 100 \% ; \mathrm{p}-$ value $_{\mathrm{P} 10 / \mathrm{P} 15}=10^{-6} ; \mathrm{p}$-value $\left.\mathrm{P}_{\mathrm{P} 15 / \mathrm{P} 60}=0.5\right)(\mathrm{Fig} .3 \mathrm{C}$ and D). We further investigated the developmental expression of MLC1 on purified GVUs. Astrocyte endfeet perivascular membranes were co-immunostained for 
Aqp4 and showed a continuous staining as previously observed (Fig. 1B). Interestingly, compared to brain slices, more MLC1-immunostained GVUs were observed at P10, and their number still increased between P10, P15 and P60 (P10:62\%; P15:80\%; P60:96\%; p-value $\mathrm{P}_{\mathrm{P} 1 / \mathrm{P} 15}=0.03$; p-value $\mathrm{P}_{\mathrm{P} / \mathrm{P} 60}=0.01$ ) (Fig. 3E and F). This difference between GVUs and slices was probably due to the fact that, even more strikingly than for GlialCAM, the shape of MLC1 staining evolved from isolated puncta on sections at P10 to clear meshworks at P15 (Fig. 3C and E). Overall, MLC1 is expressed in astrocyte perivascular endfeet after GlialCAM. It is only weakly detected before P10 and significantly increases between P10 and P15.

In the adult mouse brain, MLC1 and GlialCAM form a protein complex at the perivascular membrane (HoeggBeiler et al. 2014). We addressed the development of this complex in the GVU, analyzing the colocalization of both proteins at the gliovascular interface on hippocampal slices (Fig. 4). Since at P10 GlialCAM was more widely expressed than MLC1, we first quantified the proportion of perivascular GlialCAMpositive for MLC1 (Fig. 4). Co-localization of both proteins significantly increased after P10 (ANOVA and Bartlett's test, pvalue $=0.003$ ) and between all stages ( $\mathrm{p}$-value $\mathrm{P}_{\mathrm{P} 10 \mathrm{P} 15}=0.03 ; \mathrm{p}$-value $\left.\mathrm{P}_{\mathrm{P} 5 / \mathrm{P} 60}=0.002\right)$. While $67 \%$ of GlialCAM staining at P10 was also positive for MLC1, it represented $81 \%$ at P15 and 97\% at P60 (Fig. 4B). These results suggested the existence of two pools of GlialCAM in the developing GVUs, associated or not with MLC1, the later progressively disappearing after P10. GlialCAM was almost entirely co-localized with MLC1 at P60 as shown on GVUs (Fig. 4C). In contrast, MLC1 was systematically colocalized with GlialCAM at all stages. Altogether, our results indicate that the P10-P15 time window corresponds to a maturation step of the MLC1/GlialCAM complex in astrocyte perivascular endfeet.

We finally asked if this P10-P15 developmental window might correspond to the parallel maturation of specific brain vascular functions. The BBB is an impermeable vascular barrier, which regulates the passage of molecules between the blood and the brain. It is induced in the immature endothelium around E15 but still matures during late embryonic and postnatal development. BBB integrity depends on the expression of endothelial tight junction molecules restricting endothelial paracellular permeability and transporters (Armulik et al. 2010; Daneman et al. 2010; Tam et al. 2012; Qosa et al. 2015). Here, we characterized the expression of the tight junction protein Claudin-5 (Nitta et al. 2003), and the endothelial luminal ATP-Binding Cassette (ABC) transporter Pglycoprotein (P-gP) which effluxes xenobiotics to the blood (Schinkel et al. 1994, 1996). Strikingly, as observed for MLC1 and GlialCAM, Claudin-5 and P-gP levels detected on Western-blots of proteins extracted from purified GVUs significantly increased between P10 and P15 (Claudin-5; P5:1.0 $\pm 0.2 ;$ P10:0.8 $\pm 0.2 ;$ P15:2.8 \pm 0.3 ;

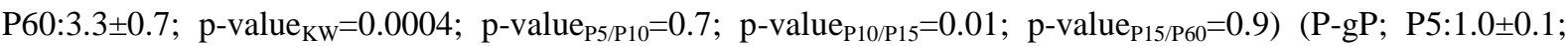




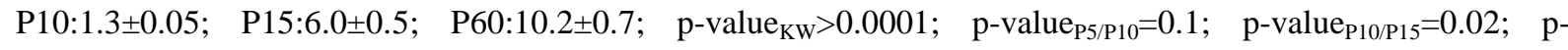
value $_{\mathrm{P} 15 / \mathrm{P} 60}=0.02$ ) (Fig. 5A and B). P-gP level continued to increase after P15. This increased expression was however not associated with a change in protein distribution within the GVU as both proteins were restricted to endothelial cells and present in all vessels (Fig. 5C). Claudin-5 signal lined endothelial cells showing the location of tight junctions. P-gP was uniformly detected in the endothelium. These results suggested a parallel maturation of the BBB and the astrocyte perivascular endfeet MLC1/GlialCAM complex between P10 and P15. 


\section{Discussion}

Our results provide the first description of the developmentally regulated perivascular expression of the MLC1/GlialCAM complex (summarized in Fig. 5C). We show that GlialCAM precedes MLC1 in the GVU and that both proteins increase within the GVU between P10 and P15, thus suggesting a progressive maturation of the MLC1/GlialCAM complex after P10. This developmental process is posterior to the formation of Aqp4 water channels in astrocyte perivascular endfeet, and parallels the maturation of the BBB characterized here by the increased expression of some key endothelial BBB components, Claudin-5 and P-gP.

We previously reported that isolating the GVU allows the characterization of the molecular properties of astrocyte endfeet and the other cellular components of the GVU, endothelial cells, pericytes and smooth muscles cells (Boulay et al. 2015a, b, c; Mazaré et al. 2018). Here, we provide the first evidence that this strategy can also be applied to characterize the GVU during brain development. In particular we determined the evolution of perivascular pool of astrocyte proteins within the GVU, which were either stable from P5 for Aqp4, or increasing between P10 and P15 for the MLC1/GlialCAM complex. Finally, the GVU purification allows to distinguish and compare the perivascular and parenchymal counterparts of astrocyte proteins as evidenced here for MLC1 which was highly enriched between P10 and P15 in the GVU fraction while constant in the brain (Fig. 3). Altogether, GVU purification is therefore a valuable tool to study astrocyte perivascular molecular properties during development.

Knowledge of astrocyte perivascular endfeet development is elusive. This question has been almost exclusively studied through the prism of Aqp4 (Lunde et al. 2015). Here, we found that most P5-vessels were covered with Aqp4-containing endfeet (Fig. 1), which confirms previous results based on brain slices showing that coverage of the brain vascular compartment by astrocyte endfeet is complete at P5 (Ezan et al. 2012). Alike Aqp4, GlialCAM, but not MLC1, appears to be another constituent of these early endfeet. Between P10 and P15, a strong increase in GlialCAM and MLC1 occurs, associated with the formation of the MLC1/GlialCAM complex detected by the colocalization of both proteins. Intriguingly, Mlc1 and Glialcam mRNA levels were stable from P5 to P15 suggesting thus that the increase of MLC1 and GlialCAM in the GVU is probably regulated by mechanisms involving post-transcriptional regulations. To further address the importance of this postnatal period in the GVU development, we examined the expression of $\mathrm{Cx} 43$, another astrocyte endfeet-enriched protein (Ezan et al. 2012) (Fig. S1). Corroborating previous observations obtained on cortical slices (Ezan et al. 2012),

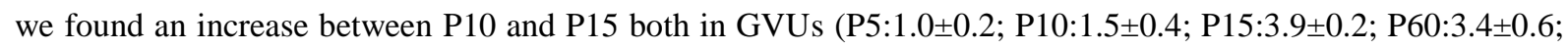




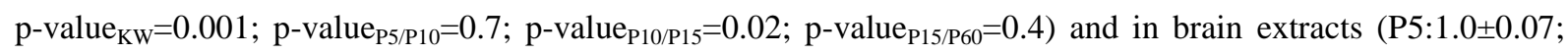

P10:1.3 $\pm 0.2 ; \quad P 15: 2.0 \pm 0.2 ; \quad P 60: 1.8 \pm 0.2 ; \quad$ p-value KW $=0.006 ; \quad$ p-value ${ }_{P 5 / P 10}=0.2 ; \quad p-$ value $_{P 10 / P 15}=0.05 ; \quad p-$ value $\left._{\mathrm{P} 15 / \mathrm{P} 60}=0.7\right)$. Altogether, these results indicated that despite the extensive Aqp4 perivascular expression at P5, astrocyte perivascular endfeet are not mature at this stage and that the P10 to P15 postnatal period could represent an important step of astrocyte endfeet maturation with the expression of $\mathrm{Cx} 43$ and the formation of the MLC1/GlialCAM complex at the junctions of astrocyte endfeet. A recent report identified a two-step maturation of astrocyte perisynaptic processes, with the formation of major branches at P7 and of finer processes ensheathing synapses at P21 (Stogsdill et al. 2017). A similar mechanism could apply to perivascular processes during postnatal development.

Our colocalization study suggested that two pools of GlialCAM might exist during the astrocyte perivascular endfeet development, with or without MLC1 (Fig.4). Since GlialCAM has been implicated in cell-matrix adhesion and cell motility processes (Chung Moh et al. 2005; Favre-Kontula et al. 2008), it might participate to the formation of astrocyte perivascular processes at P5. In contrast, from P10, GlialCAM increasingly colocalized with MLC1. In vitro and in vivo observations have shown that GlialCAM is essential for the localization of MLC1 in astrocyte membranes (López-Hernández et al. 2011b; Hoegg-Beiler et al. 2014). Similarly, Cx43 has been shown to interact with GlialCAM and to depend on GlialCAM to be correctly targeted to the membrane (Wu et al. 2016). Thus, between P10 and P15, GlialCAM might be necessary to drive the polarized localization of several astrocyte perivascular endfeet proteins.

Astrocytes are known to maintain the brain vascular properties such as BBB functions (Alvarez et al. 2013; Obermeier et al. 2013). However, astrocyte contribution during their postnatal maturation has never been documented. Our results provide evidence that the maturation of the MLC1/GlialCAM complex coincides with an increased expression of Claudin-5 and P-gP which are key endothelial proteins for BBB integrity. Thus, postnatal astrocyte endfeet and BBB maturation might be coordinated events. Interestingly, MLC patients as well as MLC mouse models with overexpression of MLC1 (Sugio et al. 2017), or deletion of MLC1 (Dubey et al. 2015) or GlialCAM (Bugiani et al. 2017) display cerebral oedema and macrocephaly during the first year of life in patients and around 3 weeks postnatal in mouse models. These data suggest that an abnormal development of the MLC1/GlialCAM complex may affect the postnatal development of the GVU resulting in impaired BBB maturation and oedema formation contributing to the MLC pathological process. MLC1/GlialCAM complex has also been shown to play a role in maintaining brain ion-water homeostasis (Ridder et al. 2011; Capdevila-Nortes 
et al. 2013b). Thus, MLC brain oedema could also be linked to an abnormal parenchymal water circulation in parallel to the Aqp4-mediated glymphatic system recently discussed (Iliff et al. 2012, 2014; Xie et al. 2013).

Altogether, our results shed new light on the gliovascular unit postnatal development. Through the study of the MLC1/GlialCAM complex, we demonstrate the existence of a progressive postnatal astrocyte endfeet molecular maturation and polarization, which might participate to the functional maturation of the gliovascular unit. 


\section{References}

Abbott NJ, Rönnbäck L, Hansson E (2006) Astrocyte-endothelial interactions at the blood-brain barrier. Nature Reviews Neuroscience 7:41-53. doi: 10.1038/nrn1824

Allen NJ, Eroglu C (2017) Cell Biology of Astrocyte-Synapse Interactions. Neuron 96:697-708. doi: 10.1016/j.neuron.2017.09.056

Alvarez JI, Katayama T, Prat A (2013) Glial influence on the blood brain barrier. Glia 61:1939-1958. doi: 10.1002/glia.22575

Amiry-Moghaddam M, Frydenlund DS, Ottersen OP (2004) Anchoring of aquaporin-4 in brain: molecular mechanisms and implications for the physiology and pathophysiology of water transport. Neuroscience 129:999-1010. doi: 10.1016/j.neuroscience.2004.08.049

Armulik A, Genové G, Mäe M, et al (2010) Pericytes regulate the blood-brain barrier. Nature 468:557-561. doi: 10.1038 /nature09522

Attwell D, Buchan AM, Charpak S, et al (2010) Glial and neuronal control of brain blood flow. Nature 468:232243. doi: 10.1038/nature09613

Ben-Zvi A, Lacoste B, Kur E, et al (2014) Mfsd2a is critical for the formation and function of the blood-brain barrier. Nature 509:507-511. doi: 10.1038/nature13324

Boulay A-C, Mazeraud A, Cisternino S, et al (2015a) Immune quiescence of the brain is set by astroglial connexin 43. J Neurosci 35:4427-4439. doi: 10.1523/JNEUROSCI.2575-14.2015

Boulay A-C, Saubaméa B, Adam N, et al (2017) Translation in astrocyte distal processes sets molecular heterogeneity at the gliovascular interface. Cell Discov 3:17005. doi: 10.1038/celldisc.2017.5

Boulay A-C, Saubaméa B, Cisternino S, et al (2015b) The Sarcoglycan complex is expressed in the cerebrovascular system and is specifically regulated by astroglial Cx30 channels. Front Cell Neurosci 9:9. doi: 10.3389/fncel.2015.00009 
Boulay A-C, Saubaméa B, Declèves X, Cohen-Salmon M (2015c) Purification of Mouse Brain Vessels. Journal of Visualized Experiments. doi: 10.3791/53208

Bugiani M, Dubey M, Breur M, et al (2017) Megalencephalic leukoencephalopathy with cysts: the Glialcam null mouse model. Annals of Clinical and Translational Neurology 4:450-465. doi: 10.1002/acn3.405

Capdevila-Nortes X, López-Hernández T, Apaja PM, et al (2013a) Insights into MLC pathogenesis: GlialCAM is an MLC1 chaperone required for proper activation of volume-regulated anion currents. Human Molecular Genetics 22:4405-4416. doi: 10.1093/hmg/ddt290

Capdevila-Nortes X, López-Hernández T, Apaja PM, et al (2013b) Insights into MLC pathogenesis: GlialCAM is an MLC1 chaperone required for proper activation of volume-regulated anion currents. Hum Mol Genet 22:4405-4416. doi: 10.1093/hmg/ddt290

Chen J, Luo Y, Hui H, et al (2017) CD146 coordinates brain endothelial cell-pericyte communication for bloodbrain barrier development. PNAS 114:E7622-E7631. doi: 10.1073/pnas.1710848114

Chung Moh M, Hoon Lee L, Shen S (2005) Cloning and characterization of hepaCAM, a novel Ig-like cell adhesion molecule suppressed in human hepatocellular carcinoma. Journal of Hepatology 42:833-841. doi: 10.1016/j.jhep.2005.01.025

Daneman R, Zhou L, Kebede AA, Barres BA (2010) Pericytes are required for blood-brain barrier integrity during embryogenesis. Nature 468:562-566. doi: 10.1038/nature09513

Dubey M, Bugiani M, Ridder MC, et al (2015) Mice with megalencephalic leukoencephalopathy with cysts: A developmental angle: MLC in Mouse and Man. Annals of Neurology 77:114-131. doi: 10.1002/ana.24307

Ezan P, André P, Cisternino S, et al (2012) Deletion of astroglial connexins weakens the blood-brain barrier. Journal of Cerebral Blood Flow \& Metabolism 32:1457-1467. doi: 10.1038/jcbfm.2012.45

Favre-Kontula L, Rolland A, Bernasconi L, et al (2008) GlialCAM, an immunoglobulin-like cell adhesion molecule is expressed in glial cells of the central nervous system. Glia 56:633-645. doi: 10.1002/glia.20640 
Ge W-P, Miyawaki A, Gage FH, et al (2012) Local generation of glia is a major astrocyte source in postnatal cortex. Nature 484:376-380. doi: 10.1038/nature10959

Hawkes CA, Härtig W, Kacza J, et al (2011) Perivascular drainage of solutes is impaired in the ageing mouse brain and in the presence of cerebral amyloid angiopathy. Acta Neuropathol 121:431-443. doi: $10.1007 / \mathrm{s} 00401-011-0801-7$

Hoegg-Beiler MB, Sirisi S, Orozco IJ, et al (2014) Disrupting MLC1 and GlialCAM and ClC-2 interactions in leukodystrophy entails glial chloride channel dysfunction. Nat Commun 5:3475. doi: 10.1038/ncomms4475

Iliff JJ, Chen MJ, Plog BA, et al (2014) Impairment of glymphatic pathway function promotes tau pathology after traumatic brain injury. J Neurosci 34:16180-16193. doi: 10.1523/JNEUROSCI.3020-14.2014

Iliff JJ, Wang M, Liao Y, et al (2012) A Paravascular Pathway Facilitates CSF Flow Through the Brain Parenchyma and the Clearance of Interstitial Solutes, Including Amyloid $\beta$. Sci Transl Med 4:147ra111147ra111. doi: 10.1126/scitranslmed.3003748

Jeworutzki E, López-Hernández T, Capdevila-Nortes X, et al (2012) GlialCAM, a Protein Defective in a Leukodystrophy, Serves as a ClC-2 Cl- Channel Auxiliary Subunit. Neuron 73:951-961. doi: 10.1016/j.neuron.2011.12.039

Jiang X, Nardelli J (2016) Cellular and molecular introduction to brain development. Neurobiology of Disease 92:3-17. doi: 10.1016/j.nbd.2015.07.007

López-Hernández T, Ridder MC, Montolio M, et al (2011a) Mutant GlialCAM Causes Megalencephalic Leukoencephalopathy with Subcortical Cysts, Benign Familial Macrocephaly, and Macrocephaly with Retardation and Autism. The American Journal of Human Genetics 88:422-432. doi: 10.1016/j.ajhg.2011.02.009

López-Hernández T, Sirisi S, Capdevila-Nortes X, et al (2011b) Molecular mechanisms of MLC1 and GLIALCAM mutations in megalencephalic leukoencephalopathy with subcortical cysts. Hum Mol Genet 20:3266-3277. doi: 10.1093/hmg/ddr238 
Lunde LK, Camassa LMA, Hoddevik EH, et al (2015) Postnatal development of the molecular complex underlying astrocyte polarization. Brain Structure and Function 220:2087-2101. doi: 10.1007/s00429014-0775-z

Mathiisen TM, Lehre KP, Danbolt NC, Ottersen OP (2010) The perivascular astroglial sheath provides a complete covering of the brain microvessels: an electron microscopic 3D reconstruction. Glia 58:10941103. doi: 10.1002/glia.20990

Mazaré N, Gilbert A, Boulay A-C, et al (2018) Connexin 30 is expressed in a subtype of mouse brain pericytes. Brain Struct Funct 223:1017-1024. doi: 10.1007/s00429-017-1562-4

Menezes MJ, McClenahan FK, Leiton CV, et al (2014) The Extracellular Matrix Protein Laminin $\alpha 2$ Regulates the Maturation and Function of the Blood-Brain Barrier. J Neurosci 34:15260-15280. doi: 10.1523/JNEUROSCI.3678-13.2014

Nagao M, Ogata T, Sawada Y, Gotoh Y (2016) Zbtb20 promotes astrocytogenesis during neocortical development. Nature Communications 7:. doi: 10.1038/ncomms11102

Nitta T, Hata M, Gotoh S, et al (2003) Size-selective loosening of the blood-brain barrier in claudin-5-deficient mice. J Cell Biol 161:653-660. doi: 10.1083/jcb.200302070

Obermeier B, Daneman R, Ransohoff RM (2013) Development, maintenance and disruption of the blood-brain barrier. Nature Medicine 19:1584-1596. doi: 10.1038/nm.3407

Qosa H, Miller DS, Pasinelli P, Trotti D (2015) Regulation of ABC efflux transporters at blood-brain barrier in health and neurological disorders. Brain Research 1628:298-316. doi: 10.1016/j.brainres.2015.07.005

Ridder MC, Boor I, Lodder JC, et al (2011) Megalencephalic leucoencephalopathy with cysts: defect in chloride currents and cell volume regulation. Brain 134:3342-3354. doi: 10.1093/brain/awr255

Sakers K, Lake AM, Khazanchi R, et al (2017) Astrocytes locally translate transcripts in their peripheral processes. PNAS 114:E3830-E3838. doi: 10.1073/pnas.1617782114 
Schinkel AH, Smit JJM, Tellingen O van, et al (1994) Disruption of the mouse mdr1a P-glycoprotein gene leads to a deficiency in the blood-brain barrier and to increased sensitivity to drugs. Cell 77:491-502. doi: $10.1016 / 0092-8674(94) 90212-7$

Schinkel AH, Wagenaar E, Mol CA, Deemter L van (1996) P-glycoprotein in the blood-brain barrier of mice influences the brain penetration and pharmacological activity of many drugs. J Clin Invest 97:25172524. doi: 10.1172/JCI118699

Schmithorst VJ, Vannest J, Lee G, et al (2015) Evidence that neurovascular coupling underlying the BOLD effect increases with age during childhood. Hum Brain Mapp 36:1-15. doi: 10.1002/hbm.22608

Schmitt A, Gofferje V, Weber M, et al (2003) The brain-specific protein MLC1 implicated in megalencephalic leukoencephalopathy with subcortical cysts is expressed in glial cells in the murine brain. Glia 44:283295. doi: 10.1002/glia.10304

Sirisi S, Folgueira M, López-Hernández T, et al (2014) Megalencephalic leukoencephalopathy with subcortical cysts protein 1 regulates glial surface localization of GLIALCAM from fish to humans. Hum Mol Genet 23:5069-5086. doi: 10.1093/hmg/ddu231

Stogsdill JA, Ramirez J, Liu D, et al (2017) Astrocytic neuroligins control astrocyte morphogenesis and synaptogenesis. Nature 551:192-197. doi: 10.1038/nature24638

Sugio S, Tohyama K, Oku S, et al (2017) Astrocyte-mediated infantile-onset leukoencephalopathy mouse model. Glia 65:150-168. doi: 10.1002/glia.23084

Tam SJ, Richmond DL, Kaminker JS, et al (2012) Death Receptors DR6 and TROY Regulate Brain Vascular Development. Developmental Cell 22:403-417. doi: 10.1016/j.devcel.2011.11.018

Teijido O, Casaroli-Marano R, Kharkovets T, et al (2007) Expression patterns of MLC1 protein in the central and peripheral nervous systems. Neurobiology of Disease 26:532-545. doi: 10.1016/j.nbd.2007.01.016

van der Knaap MS, Boor I, Estévez R (2012) Megalencephalic leukoencephalopathy with subcortical cysts: chronic white matter oedema due to a defect in brain ion and water homoeostasis. The Lancet Neurology 11:973-985. doi: 10.1016/S1474-4422(12)70192-8 
Vasile F, Dossi E, Rouach N (2017) Human astrocytes: structure and functions in the healthy brain. Brain Struct Funct 222:2017-2029. doi: 10.1007/s00429-017-1383-5

Wu M, Moh MC, Schwarz H (2016) HepaCAM associates with connexin 43 and enhances its localization in cellular junctions. Sci Rep 6:36218. doi: 10.1038/srep36218

Xie L, Kang H, Xu Q, et al (2013) Sleep Drives Metabolite Clearance from the Adult Brain. Science 342:373377. doi: $10.1126 /$ science. 1241224

Yousif S, Marie-Claire C, Roux F, et al (2007) Expression of drug transporters at the blood-brain barrier using an optimized isolated rat brain microvessel strategy. Brain Research 1134:1-11. doi: 10.1016/j.brainres.2006.11.089 


\section{Figure legends}

\section{Figure 1: Purification of gliovascular units from postnatal developing brains}

A. Schematic representation of the gliovascular unit (GVU) purification process indicating its cellular components. A, astrocyte; AE, astrocyte endfeet; E, endothelial cell; M, mural cell; L, vessel lumen;. An astrocyte is shown in red. B. Confocal projection images of purified GVUs from P5 and P60 brains immunolabelled for Aqp4 (red) showing that astrocyte perivascular membranes are co-purified with brain vessels as early as P5. Nuclei are labelled with Hoechst (blue) and blood vessel walls with IB4 (grey). C. Quantification of the proportion of Aqp4-positive GVU segments between P5 and P60. Most purified GVUs contain astrocyte Aqp4-positive perivascular membranes. This proportion does not change after P5. D, E. Western-blot analysis (D) and quantification (E) of whole brain- and GVU-purified proteins between P5 and P60. $\beta$-actin is used as a loading control. The level of Aqp4 in purified GVUs and brain remains constant during postnatal development. ns, p>0.05. GVU, gliovascular unit; IB4, Isolectin GS-B4.

\section{Figure 2: Postnatal expression of GlialCAM in astrocyte perivascular endfeet}

A.Western-blot analysis and quantification of whole brain- and GVU-purified proteins between P5 and P60. $\beta$ actin is used as a loading control. GlialCAM expression significantly increases between P10 and P15 in the purified GVUs and between P5 and P10 in the brain. B. qPCR analysis of whole brain mRNAs between P5 and P60. Gapdh is used as a loading control. GlialCAM expression significantly increases at P60. .. C-F. Projection confocal images and quantification of GlialCAM-immunolabelled vessels (green) in hippocampal slices (C, D) and purified GVUs (E, F) at P10 and P15. Nuclei are labelled with Hoechst (grey) and blood vessel wall with IB4 (red). GlialCAM is present around a majority of brain vessels. *, p<0.05; ns, p $>0.05$. WB, Western Blot; GVU, gliovascular unit; IB4, Isolectin GS-B4.

\section{Figure 3: Postnatal expression of MLC1 in astrocyte perivascular endfeet}

A.Western-blot analysis and quantification of whole brain- and GVU-purified proteins between P5 and P60. $\beta$ actin is used as a loading control. MLC1 expression significantly increases between P10 and P15 in the purified GVUs but not in the brain. B. qPCR analysis of whole brain mRNAs between P5 and P60. Gapdh is used as a loading control. MLC1 expression is constant. C-F. Projection images and quantification of MLC1immunolabelled vessels (green) in hippocampal slices (C, D) and purified GVU (E, F) at P10 and P15. Nuclei are labelled with Hoechst (grey), blood vessel wall with IB4 (red) and astrocyte endfeet are immunolabelled for 
Aqp4 (red). MLC1-positive vessel proportion increases mainly between P10 and P15. ***, p<0.001; *, p<0.05; ns, p>0.05. WB, Western Blot; GVU, gliovascular unit; IB4, Isolectin GS-B4.

\section{Figure 4: Postnatal maturation of the astrocyte endfeet MLC1/GlialCAM complex}

A. Representative projection image of P10 brain slices immunolabelled for MLC1 (green) and GlialCAM (red) showing GlialCAM colocalized with MLC1 (arrow) or not (arrowhead). Blood vessel wall are labelled with IB4 (grey). B. Quantification of perivascular GlialCAM colocalized with MLC1 (black) and perivascular MLC1 colocalized with GlialCAM (white). Colocalization of GlialCAM with MLC1 increases significantly after P10 while MLC1 is always colocalized with GlialCAM. C. Projection image of a P60-purified GVU immunolabelled for MLC1 (green) and GlialCAM (red) showing extensive colocalization into networks delimiting astrocyte endfeet. Nuclei are labelled with Hoechst (blue) and blood vessel wall with IB4 (grey).**, p<0.01 *, p<0.05; ns, p>0.05. GVU, gliovascular unit; IB4, Isolectin GS-B4.

\section{Figure 5: P10-P15, a temporal window for the GVU maturation.}

A, B. Western-blot analysis (A) and quantification (B) of the tight junction protein Claudin-5 and the $A B C$ transporter P-Glycoprotein (P-gP), on protein extracts from purified GVUs between P5 and P60. $\beta$-actin is used as a loading control. Both proteins increase significantly between P10 and P15 and P60 for P-gP. C. Representative projection image of P10, P15 and P60-purified GVU immunolabelled for P-gP (green) and Claudin-5 (red). Nuclei are labelled with Hoechst (blue) and blood vessel wall with IB4 (grey). D. Visual abstract of the results outlining the changes observed between P10, P15 and P60 within the GVU. At P10, Aqp4 (dark blue) is present in all endfeet, GlialCAM (red) in almost all endfeet, while MLC1 (green) is restricted to some endfeet. Both MLC1 and GlialCAM increase significantly within GVU between P10 and P15. This increase is associated with a progressive colocalization of both proteins (yellow), indicating a maturation of the MLC1/GlialCAM complex at the junctions between endfeet. This maturation of MLC1/GlialCAM correlates with an increased expression of endothelial proteins Claudin-5 (light blue) and P-gP (black) which participate to the BBB formation. *, $\mathrm{p}<0.05 ; \mathrm{ns}, \mathrm{p}>0.05$. GVU, gliovascular unit.

\section{Figure S1: Postnatal expression of Connexin 43 in astrocyte perivascular endfeet}

Western-blot analysis (A) and quantification (B) of Connexin 43 (Cx43) expression in purified GVU and whole brains and between P5 and P60. $\beta$-actin is used as a loading control. The variable presence of the two upper Cx43 phosphorylated bands in the P60 samples is linked to the migration protocol. Cx43 increases between P10 and P15 both in GVUs and brain. *, p<0.05; ns, p>0.05. GVU, gliovascular unit. 


\section{Figure S2: Detailed Western Blot results}

Detailed Western-Blot analysis presented in the figures. Squared areas indicate the bands analyzed for quantification. Saturated signal present on the MLC1 (GVU) Western-Blot is due to rehybridation of the membrane with $\beta$-actin. GVU, gliovascular unit. 